\section{SOBRE EL REALISMO MATEMÁTICO DE ZUBIRI Y SU INTERPRETACIÓN DE LOS TEOREMAS DE GÖDEL Y COHEN}

\author{
Oscar Orellana Estay \\ Universidad Técnica Federico Santa María, Chile \\ oscar.orellana@usm.cl \\ Ronald Durán Allimant \\ Pontificia Universidad Católica de Valparaíso, Chile \\ Universidad de Playa Ancha, Chile \\ ronald.duran@upla.cl
}

Cómo citar este artículo/Citation: Orellana Estay, O. y Durán Allimant, R. (2016). Sobre el realismo matemático de Zubiri y su interpretación de los teoremas de Gödel y Cohen. Arbor, 192 (780): a333. doi: http://dx.doi.org/10.3989/ arbor.2016.780n4009

Recibido: 25 julio 2014. Aceptado: 01 septiembre 2015.

RESUMEN: En este artículo se analiza el apéndice «La realidad de lo matemático» del libro Inteligencia y Logos, del filósofo español Xavier Zubiri, con el objetivo de clarificar su concepción de los objetos matemáticos como reales por construcción, y a partir de esto su interpretación de los teoremas de Gödel y Cohen, que según Zubiri dejarían en evidencia que la realidad de los objetos matemáticos es anterior a su verdad. Para ello se expone primero, la concepción de Zubiri de los objetos matemáticos; luego se distingue su posición de la de los formalistas e intuicionistas; por último, se analiza la interpretación zubiriana de los teoremas de Cohen y Gödel.

PALABRAS CLAVE: Zubiri; realidad; matemático; construcción; Cohen; Gödel.

\section{ON ZUBIRI'S MATHEMATICAL REALISM AND HIS INTERPRETATION OF GÖDEL'S AND COHEN'S THEOREMS}

Copyright: (C) 2016 CSIC. Este es un artículo de acceso abierto distribuido bajo los términos de la licencia Creative Commons Attribution (CC BY) España 3.0.

ABSTRACT: In this article we analyze the appendix «La realidad de lo matemático» [The reality of the mathematical], of the Spanish philosopher's book Inteligencia y Logos, with the aim of clarifying his concept of mathematical objects as real by construction, and his interpretation of Gödel's and Cohen's theorems, both of which would show that the reality of mathematical objects is previous to their truth. To do this, we first present Zubiri's concept of mathematical objects; then we distinguish his position from that of the formalists and intuitionists; and finally, we analyze a Zubirian interpretation of Gödel's and Cohen's theorems.

KEYWORDS: Zubiri; reality; mathematical; construction; Cohen; Gödel. 


\section{INTRODUCCIÓN}

En el presente artículo se analiza el apéndice "La realidad de lo matemático» que aparece en Inteligencia y Logos (Zubiri, 1982, pp. 133-146) para clarificar la posición de Zubiri respecto a las matemáticas. En dicho apéndice se estudia, por así decir, el estatuto o naturaleza "ontológica» de los objetos matemáticos. En filosofía de las matemáticas es usual preguntarse: ¿qué tipo de objetos son los objetos matemáticos?, ¿son estos objetos ideales, reales, ficticios, formales, convencionales, lógicos, arbitrarios, abstractos, estructurales, mentales, nominales, etc.? Zubiri nos dirá que los objetos matemáticos son reales por postulación, por construcción, a partir de lo cual interpretará los teoremas de Gödel y Cohen, estableciendo que éstos dejan en evidencia que la realidad de los objetos matemáticos es anterior a su verdad. En lo que sigue, analizaremos y trataremos de clarificar estas afirmaciones. Para ello seguiremos el siguiente plan: en primer lugar, se expone la idea de Zubiri de la realidad de los objetos matemáticos como construcción; luego, se distingue esta posición respecto a la de los formalistas e intuicionistas; y finalmente, se analiza y precisa la interpretación zubiriana de los teoremas de Cohen y Gödel, matizando las implicaciones derivadas por el filósofo español.

\section{LA MATEMÁTICA COMO SISTEMA DE JUICIOS SOBRE OBJETOS REALES}

Penelope Maddy en su libro Realism in Mathematics dice: "Los matemáticos, aunque están al tanto de un rango de verdades matemáticas más amplio que el de muchos de nosotros, a menudo se inclinan a estar de acuerdo con el impoluto sentido común respecto a la naturaleza de aquellas verdades. Se ven a sí mismos y a sus colegas como investigadores descubriendo las propiedades de varios distritos fascinantes de la realidad matemática: los teóricos de los números estudian los enteros, los geómetras estudian ciertos espacios de buen comportamiento, los teóricos de grupos estudian grupos, los conjuntistas conjuntos, y así» (Maddy, 1990, p. 1) ${ }^{1}$. Sin embargo, cuando se les presiona un poco a los matemáticos preguntando: ¿dónde se encuentran los objetos matemáticos?, ¿cuál es la naturaleza de estos objetos?, etc., la mayoría de ellos retroceden y se refugian en un formalismo vacío o un idealismo prehistórico. De acuerdo a esta última postura es usual llamar a los objetos matemáticos: «objetos ideales». Pues bien, para Zubiri los objetos matemáticos no son ideales, sino que reales.
Según Zubiri: "La matemática no es un sistema de verdades necesarias, y meramente coherentes entre sí de acuerdo con los "principios" de la lógica, sino que es un sistema de verdades necesarias acerca de un objeto que, a su modo, tiene realidad ante la inteligencia» (Zubiri, 1982, p. 129). La matemática no es un mero juego lógico-formal de símbolos sin significado alguno, como lo es para los formalistas puros, sino que es un sistema de verdades necesarias acerca de un objeto que es sentido físicamente (sentientemente) en la misma realidad física en que es real una piedra. "Sin sentir lo matemático, no se puede construir la matemática» (Zubiri, 1982, p. 145). La matemática está compuesta por juicios ${ }^{2}$ acerca de objetos reales, los objetos matemáticos, y no meramente de proposiciones, enunciados, o sentencias lógico-formales, como es común pensar desde una perspectiva logicista. Dice Zubiri: "la matemática está compuesta de juicios que recaen sobre algo que es real por postulación» (Zubiri, 1982, p. 133). El juicio es juicio de algo real. En el caso de las matemáticas, se juzga acerca de objetos reales por postulación: "todo juicio, toda afirmación, lo es de algo real presupuesto como tal a la afirmación misma. Cuando las cosas son reales en y por sí mismas [por ejemplo una piedra], aquella presuposición es formalmente la aprehensión primordial de realidad. Cuando las cosas son reales, pero realizadas constructivamente [por ejemplo, los objetos matemáticos o figuras de ficción], entonces la presuposición es formalmente postulación. La postulación es posible sólo por estar intrínseca y formalmente fundada en la aprehensión primordial de realidad» (Zubiri, 1982, pp. 131-132). Como queda claro de lo anterior, el hecho de que Zubiri hable de juicios no significa que hace de la matemática una ciencia subjetiva, que tratara de objetos subjetivos, o cuyo fundamento fuera subjetivo, sino que: "la estructura primaria y radical del juicio es ser una afirmación de una cosa aprehendida ya como real (en aprehensión primordial) pero según su momento formalmente campal» (Zubiri, 1982, p. 132). En consecuencia, el juicio presupone la aprehensión primordial de realidad, y por tanto, la realidad de aquello de que se juzga, pero según su momento formalmente campal. El juicio no es intelección inmediata de algo real, sino que: «[el juicio]es una intelección modalizada de aquella aprehensión, de aquella intelección directa e inmediata [...] no se trata de una presuposición de índole procesual, es decir no se trata de que antes de juzgar se aprehende realidad, sino de que esta realidad 
aprehendida antes de juzgar se mantiene como momento formalmente constitutivo del juicio mismo en cuanto tal» (Zubiri, 1982, p. 132). Queda claro que no se está "subjetivando» cuando se habla de que la matemática está compuesta de juicios.

Ahora bien, Zubiri precisa lo propio de los juicios matemáticos, puesto que no se refieren a "cosasrealidad»: "Se dirá que hay muchísimos juicios que no están en este caso [el referirse a cosas-realidad, es decir, juicios sobre cosas como sillas o mesas, en tanto reales] porque recaen sobre cosas que no son reales: es el caso de todos los juicios de la matemática, y también de los innumerables juicios que intervienen en un relato de ficción, por ejemplo en una novela [...] Es cierto que un espacio geométrico o Don Juan no son cosas reales en la misma forma en que lo es un vaso de agua. Pero ¿funcionan por así decirlo como algo pura y simplemente no real? De ninguna manera» (Zubiri, 1982, p. 128). El espacio geométrico, por ejemplo, no siendo un espacio físico (sensualmente hablando), no se reduce a un mero concepto, ni a una síntesis de conceptos. Si fuera así, ese espacio no pasaría de ser lo que el espacio «sería» (Zubiri, 1982, p. 128). Sin embargo, la matemática, o mejor dicho los matemáticos no hablan, ni tratan de espacios que "serían», sino que de espacios que «son». "Esto significa que los conceptos, simples aprehensiones de lo que los espacios "serían", se tornan en conceptos de algo que "es" » (Zubiri, 1982, p. 128). Y ¿cómo ocurre semejante cosa?, es decir, ¿cómo algo que "sería» se transforma en algo que «es»? Pues bien, en el caso de las matemáticas, los meros conceptos se tornan en conceptos de algo que "es» en virtud de un sistema de postulados.

¿Qué es lo que los postulados postulan? Al modo de ver de Zubiri los postulados no postulan «verdad» sino que realidad, no piden que se admita meramente su verdad (ya sea por su evidencia o alguna otra razón). Si así fuera, la matemática se reduciría a la lógica. Esta fue la posición logicista que adoptaron Frege, Russell, Whitehead, entre otros matemáticos y filósofos, que participaron y vivieron la crisis de los fundamentos de las matemáticas a finales del siglo XIX y principios del XX. Zubiri aclara más aún su posición: «los postulados no son meros enunciados lógicos sino enunciados de los caracteres que tiene el "contenido" de la "realidad" de lo postulado. La "postulación" se funda en el "sería" y consiste formalmente en su transformación en "es" gracias a la postulación de la realidad [...] es formalmente construcción» (Zubiri, 1982, p. 129). Es lo que veremos en el siguiente acápite.

\section{LA POSTULACIÓN DE REALIDAD EN MATEMÁTICAS COMO CONSTRUCCIÓN}

La integral, la derivada, el espacio geométrico, etc., no son sentidos sensualmente, no tenemos registros sensibles de ellos, pero para Zubiri son reales. "No se trata de que un espacio geométrico o un número irracional sean sentidos como se siente un color; esos objetos evidentemente no son sensibles. Se trata de que el modo de intelección de un número irracional o de un espacio geométrico es sentiente. $\mathrm{Y}$ lo es: $1^{\circ}$ porque se intelige postuladamente en un campo de realidad, esto es en la formalidad dada en impresión de realidad, y $2^{\circ}$ porque su construcción misma no es mera conceptuación sino realización, es decir algo llevado a cabo sentientemente. Sin sentir lo matemático, no se puede construir la matemática» (Zubiri, 1982, p. 145). Los objetos matemáticos son reales y no meramente conceptivos, su contenido está realizado, proyectado en «la» realidad y son inteligidos sentientemente. Los contenidos de los objetos matemáticos son postulados en un campo de realidad que es el mismo que el de una piedra. La diferencia entre ellos concierne tan sólo al contenido, en efecto: en el primer caso, el contenido es libremente postulado en la realidad, mientras que en el segundo caso el contenido está dado en la realidad. Por lo tanto, nos dice Zubiri: «los objetos matemáticos no tienen existencia ideal sino solamente existencia postulada, postulada pero en "la" realidad. Lo que sucede es que su contenido: $1^{\circ}$ está construido, y $2^{\circ}$ lo está según conceptos. Lo que tan impropiamente se llama ideal es lo real construido según conceptos» (Zubiri, 1982, p. 144).

La postulación matemática no es meramente conceptual, sino que es "realización», es construcción. "La realización misma puede y debe llamarse construcción» (Zubiri, 1982, p. 138). La construcción matemática, según Zubiri realiza postuladamente estos conceptos objetivos, al asignarle libremente por postulación ciertas notas o propiedades, existencia y contenido, y proyectar tales contenidos sobre «la» realidad (Zubiri, 1982, pp. 136-138). Este modo propio de postulación de realidad de la matemática, proyecta lo irreal del concepto sobre «la» realidad "según conceptos» (a diferencia de la postulación de la novela, por ejemplo, que lo hace mediante fictos) (Zubiri, 1982, pp. 128-132) ${ }^{3}$. Así: «construcción es un modo de realización: es realizar según conceptos» (Zubiri, 1982, pp. 136-137). Podríamos decir que por un lado están los conceptos matemáticos con sus aspectos irreales y por otro «la» realidad, y es el modo matemático de postular, la construcción matemática, 
la que realiza estos conceptos proyectando sus contenidos sobre «la» realidad. Entonces, es la construcción matemática o el modo matemático de postular la que, por así decir, hace "el milagro» de volver reales los contenidos de los conceptos objetivos, en el sentido de que al proyectarlos "según conceptos» sobre «la» realidad son sentidos en aprehensión primordial y tratados como «son», en lugar de como "serían». Los objetos matemáticos no son sentidos en aprehensión primordial porque ya estén en la formalidad de realidad, sino que son proyectados conceptualmente sobre la formalidad de realidad.

Estos objetos matemáticos reales, con propiedades y notas bastante precisas, son investigados, tratados, manipulados, usados, etc., en virtud de los contenidos reales postulados, construidos. A partir de las notas o propiedades, existencia y contenidos realizados por postulación, se pueden establecer (deducir), la realización de otras notas, sin salir de lo real por postulación. Ahora bien, lo real por postulación por ser real tiene más notas, o propiedades, que las postuladas, y que las que se puedan deducir de los postulados. Lo postulado por ser postulado en la realidad "tiene más propiedades que las formalmente enunciadas en un principio» (Zubiri, 1982, p. 129). Zubiri nos dice: «la actualización de "la" realidad en la intelección deja libre su contenido. Y entonces lo que el postulado postula es que tal contenido determinado (por ejemplo, el paralelismo euclidiano o la topología no-arquimediana), tanto en sus notas como en su existencia, es el que se realiza en "la" realidad, en el "más", en esta misma realidad física según la cual es real esta piedra. Este contenido así realizado es, según hemos dicho, "cosa libre". El espacio geométrico es real con la misma realidad según la cual es real esta piedra. No es un mero concepto, pero es realidad libremente realizada: libre pero real, real pero libre. Esta postulación postula por tanto que "la" realidad se realiza en tal contenido: se postula esta realización» (Zubiri, 1982, p. 136). La libre creación que se practica en el proceso de creación de contenido, para obtener un concepto objetivo, no es sensible, es propio de una inteligencia sentiente, y el objeto matemático es una realidad propia, realidad física impresivamente sentida en aprehensión primordial: «Sólo una inteligencia sentiente puede por ejemplo no sentir el contenido de un conjunto continuo, esto es el conjunto de los números irracionales, y sin embargo realizar libremente este contenido (conceptuado sea por meras definiciones, sea por operaciones ejecutadas) de un modo sentiente» (Zubiri, 1982, pp. 142-143). Esto es construcción según Zubiri.
Los objetos así construidos son reales, son construidos en la misma realidad que la de una piedra, a saber: en la formalidad de realidad presente en la aprehensión primordial; sólo que no son reales en y por sí mismos como la piedra, porque esta posee contenidos sensualmente detectables y medibles, mientras que los objetos matemáticos tienen notas o propiedades, existencia y contenidos por postulación, los cuales se proyectan sobre «la» realidad. Por esto, nos dice Zubiri: «el objeto así construido [el objeto matemático] es una estricta realidad que puede tener propiedades o notas "suyas", "propias", y no sólo propiedades "deducidas" de los axiomas y postulados» (Zubiri, 1982, p. 138). Una cosa son las propiedades deducidas y otras son las propiedades que ya están formalmente en el objeto, y los objetos matemáticos por ser reales tienen propiedades que les pertenecen «de suyo». Pareciera entonces que de acuerdo a Zubiri, los objetos matemáticos tienen dos tipos de propiedades, las deducidas y las «suyas». A reglón seguido Zubiri nos dice: «Es que el objeto real postuladamente realizado según conceptos tiene, por estar realizado, más notas o propiedades que las definidas en su postulación» (Zubiri, 1982, p. 138). Ahora bien: ¿existirán objetos matemáticos cuyas notas y propiedades sean todas definibles por postulación? La respuesta es sí. Existen varios sistemas axiomáticos consistentes y completos, como por ejemplo: el cálculo de predicados de primer orden y la geometría de Hilbert. Es decir, de acuerdo a esto, existen objetos matemáticos cuyas notas y propiedades se agotan completamente en el sistema axiomático que los formaliza. Veremos esto con más detalles más adelante al hablar sobre la interpretación zubiriana de los teoremas de Gödel y Cohen.

Resumiendo, un objeto matemático no es real por su mera definición dada a través de un sistema de axiomas a la Hilbert, tampoco es un objeto real porque se ejecute a través de un número finito de operaciones o pasos a la Brouwer, y tampoco es un objeto real en y por sí mismo. Los objetos matemáticos son reales debido a una postulación que realiza sus contenidos (notas o propiedades y existencia, libremente determinadas gracias a la postulación ante la inteligencia), en la formalidad de realidad aprehendida en aprehensión primordial. En consecuencia, los objetos matemáticos no son ideales.

\section{EL MÉTODO AXIOMÁTICO Y EL FORMALISMO EN MA- TEMÁTICAS}

Antes de seguir, tenemos que decir algo respecto al método axiomático y el formalismo en matemáticas, y la idea de construcción asociada a ellos. 
En matemáticas, la noción de "construcción» está asociada al método axiomático, sin ser, sin embargo, éste el único método que esta ciencia emplea. El método axiomático emerge con Euclides (300 A.C.) y la geometría que él formalizó, a través de un cierto número de definiciones, principios y postulados. Este método se retoma en la primera mitad del siglo pasado debido a la crisis en los fundamentos de las matemáticas, la cual fue provocada por una serie de paradojas, como por ejemplo: la paradoja de Russell y la paradoja de Burali-Forti. Una de las reacciones a esta crisis consistió en no insistir en la utilización de definiciones intuitivas y/o por género próximo y diferencia específica. En lugar de ello se optó por considerar los objetos matemáticos como primitivas sin definirlos (para ser enfáticamente redundante), cuyas reglas de juego (la sintaxis o forma de operar entre ellos), quedaban expresadas por medio de axiomas o postulados o principios. A este método se le llamó método axiomático, y al movimiento fundacional que lo cultivó y lo defendió se le llamó formalismo hilbertiano, debido a que fue David Hilbert (matemático alemán muy influyente de la primera mitad del siglo pasado) quien lo propuso y difundió originalmente. A la sazón, también existen los que podríamos llamar métodos de construcción logicista y el método de construcción intuicionista, por ello Zubiri se ve en la obligación de hacer la diferencia entre lo que él entiende por construcción y la construcción en el sentido de Gödel y Brouwer, respectivamente, como veremos más adelante.

Básicamente existen dos versiones del formalismo, que equivalen a dos formas de hablar ligeramente diferentes. (1) Algunos formalistas sostienen que las afirmaciones o proposiciones matemáticas pueden pensarse como afirmaciones o proposiciones acerca de ciertas reglas de manipulación de secuencias de símbolos. Por ejemplo, en la Geometría Euclidiana (la cual consiste en ciertas secuencias Ilamadas axiomas y algunas reglas de inferencia que permiten generar nuevas secuencias a partir de las dadas) se puede probar que el Teorema de Pitágoras es válido (es decir, se puede generar la secuencia correspondiente al Teorema de Pitágoras). Entonces, de acuerdo a esta forma de hablar, las verdades matemáticas no son acerca de números, conjuntos, triángulos, etc., no son acerca de nada en absoluto, son simplemente un juego simbólico puramente formal, sin ningún significado. (2) La otra versión se conoce como deductivismo. De acuerdo al deductivismo, el Teorema de Pitágoras no es una verdad absoluta, sino una verdad relativa, en el sentido de que si se le asignan significados a las secuencias de símbolos de manera que, las reglas del juego son verdaderas (es decir, si asignamos afirmaciones o proposiciones verdaderas a los axiomas y las reglas de inferencia preservan la verdad), entonces debemos aceptar la verdad de los teoremas, o mejor dicho debemos aceptar que de acuerdo a la interpretación que le hemos dado a los axiomas, las secuencias de símbolos deducidas son afirmaciones o proposiciones verdaderas. Lo mismo se sostiene para cualquier otra afirmación o proposición matemática. Entonces, de acuerdo a esta versión del formalismo las matemáticas no necesariamente son un juego simbólico sin significado alguno. Pero, usualmente, la tarea o problema de interpretar el sistema axiomático en cuestión se deja a los filósofos o científicos. Es decir, la tarea de construir o encontrar un modelo del sistema axiomático, de acuerdo al cual las secuencias de símbolos llamadas axiomas y las que se deduzcan de ellas por medio de reglas de inferencia válidas, tengan una interpretación no se considera tarea del formalista.

Un ejemplo sencillo de teoría abstracta es el siguiente:

AXIOMA 1. Entre ciertos elementos $P$ y ciertos elementos $r$ existe una relación "determinada» tal que: Dos $\mathrm{P}$ (P1 y P2) determinan un r, y sólo uno. En tal caso: DEFINICIÓN 1. Diremos que P1 y P2 pertenecen a $r$, o que $r$ pasa por P1 y $\mathrm{P} 2$.

AXIOMA 2. Existe por lo menos un P.

AXIOMA 3. A cada $r$ pertenecen por lo menos dos $P$ distintos.

AXIOMA 4. Por cada P pasan por lo menos dos $r$ distintos.

TEOREMA 1. Existen (por lo menos) tres P distintos.

DEMOSTRACION: Deben aplicarse sucesivamente los axiomas 2, 4, 3 y 1.

Ahora, daremos algunas interpretaciones diferentes de esta teoría abstracta:

P son los puntos y $r$ son las rectas en la geometría del espacio.

P son las rectas y $r$ son los puntos en la geometría del espacio.

P son las circunferencias de un plano y $r$ los haces de circunferencias (conjunto de circunferencias tales, que dos a dos tienen el mismo eje radical).

P y $r$ son los vértices y lados de un triángulo.

P y $r$ son los vértices y aristas de un tetraedro.

etc. 
Es pertinente hacer notar que no debemos confundir los diferentes métodos que se utilizan en matemáticas para demostrar teoremas, lemas y corolarios (como por ejemplo: reducción al absurdo, modus ponens, inducción completa, método directo, indirecto, contra positivo, reducción, análisis, genético, etc.), con los métodos que usan las posturas filosóficas formalistas, logicistas e intuicionistas para sustentar sus posiciones argumentativas, es decir: no se debe confundir la matemática con la meta-matemática; no se deben confundir los métodos locales (técnicos), con los métodos globales (reflexivos); no se deben confundir los métodos endógenos con los métodos "exógenos»; aunque sea difícil separarlos claramente, porque se traslapan y se usan indistintamente todos ellos tanto en la actividad matemática propiamente tal, como en la meta-matemática. Aclaramos esto aquí, porque obviamente Zubiri no está tan interesado en los detalles de la actividad matemática propiamente tal, sino más bien en la matemática como un todo.

Sin embargo, en relación con esto, es conveniente dejar aquí consignada la pregunta: ¿se pueden, o mejor dicho se deben considerar todos los objetos matemáticos como una construcción, como una postulación de realidad, como al parecer propone Zubiri; o los objetos matemáticos son descubiertos (indirectamente si se quiere), porque forman la estructura básica de la realidad; o tan sólo son un invento una ficción útil, como piensa Hartry Field? Por cierto, la matemática tiene mucho de construcción en el sentido tanto zubiriano (como en otros sentidos), a nivel conceptual (a nivel de lenguaje simbólico), pero cuando, por ejemplo, uno mira el "Conjunto de Julia" (conjuntos fractales) es casi imposible no preguntarse si acaso este conjunto ya no estaba allí desde el comienzo y sólo ahora lo vinimos a descubrir, describir y representar en nuestro lenguaje (como parecen probar todos los ejemplos de fractales que se encuentran en la naturaleza), o cuando examinamos algunas propiedades extrañísimas de los Números Primos, las cuales parecen no tener relación alguna con la realidad, sino que más bien son la creación de una mente delirante.

Volviendo al tema de la construcción, Zubiri distingue su concepto de construcción del de Gödel y Cohen, y del de Brower tal como veremos a continuación en las secciones siguientes.

\section{LA CONSTRUCCIÓN EN EL SENTIDO DE ZUBIRI V/S LA CONSTRUCCIÓN EN EL SENTIDO DE GÖDEL Y COHEN (AXIOMATISMO FORMALISTA)}

Según Zubiri: «Gödel llama construir un conjunto a generarlo según la aplicación iterada de ciertas opera- ciones axiomáticamente definidas en los axiomas de Zermelo-Fraenkel [Z-F]» (Zubiri, 1982, p. 137), poniendo el acento en operaciones definidas como tales. En consecuencia, no se trataría del procedimiento para llevar a cabo tales operaciones. En otras palabras, Gödel también construye, en este caso conjuntos, pero lo hace aplicando iteradamente operaciones definidas en los axiomas de Z-F, sin presentar la manera, o forma, o algoritmo para hacer las operaciones mismas. Es decir, se trata de la aplicación de operaciones definidas axiomáticamente, pero no procesualmente, porque las definiciones axiomáticas no muestran ni describen el procedimiento (el algoritmo de desenvolvimiento de la operación). Por ejemplo, la unión de conjuntos esta definida en los axiomas de Z-F de la siguiente manera (dicho en palabras): «Axioma de las uniones: Para toda colección de conjuntos existe un conjunto que contiene a todos los elementos que pertenecen cuando menos a uno de los conjuntos de la colección», pero no se dice cómo se hace la unión de dos conjuntos dados. Esto requiere la descripción de un procedimiento, por lo demás bastante trivial, a saber: dados dos conjuntos, la unión de ellos consiste no sólo en considerar, sino que en colocar, los elementos de ambos conjuntos en uno solo, eliminando repeticiones.

Los conjuntos generados por la aplicación iterada de operaciones axiomáticamente definidas en los axiomas de Z-F, son los que, según Zubiri, Gödel Ilamó «construibles». Supuestamente, Cohen (quien fuera discípulo de Gödel), se apoyó en conjuntos noconstruibles, en este sentido, para probar en 1963 que la negación de la hipótesis del continuo de Cantor (HCC) era consistente con los axiomas de Z-F, 25 años después de que Gödel (su maestro) probara que la hipótesis del continuo de Cantor era consistente con los axiomas de Z-F, todo lo cual deja en claro que la hipótesis del continuo de Cantor es una proposición indecidible en el contexto de los axiomas de Z-F, es decir, no es ni verdadera, ni falsa, por de pronto.

Ahora bien, Zubiri nos hace notar que los elementos de todo conjunto (construible o no construible, en el sentido de Gödel y Cohen, respectivamente), tienen dos clases de propiedades, a saber: específicas y genéricas. Las específicas, nos dice Zubiri, son: «las que responden a los postulados y axiomas operacionales a que acabo de referirme» (Zubiri, 1982, p. 137), es decir, aquellas propiedades que están implícitas en las operaciones definidas axiomáticamente. Las genéricas, nos dice Zubiri, son aquellas: «en virtud de las cuales forman un conjunto [los elementos] dejando indeterminadas las propiedades específicas, las cua- 
les [a su vez] "forzarán" a las propiedades genéricas a especificarse» (Zubiri, 1982, p. 137). Así las cosas, las propiedades específicas son las que nos permiten obtener conjuntos construibles en el sentido de Gödel, mientras que las genéricas sólo nos permiten obtener conjuntos no construibles en el sentido de Cohen. Las propiedades específicas son las que forzarán a las propiedades genéricas a especificarse. Para mayor abundamiento, note que aquí lo genérico se opone a lo específico, tal vez en el sentido de género próximo y diferencia específica. En efecto, algunos autores, para evitar antinomias (es decir, expresiones contradictorias), consideraron que solamente puede formarse un conjunto con objetos que existan anteriormente (tarea de los axiomas y postulados). Así, definir un conjunto, digamos $C$, es dar una propiedad característica, digamos $\mathrm{P}$, de ciertos elementos de un conjunto $U$, anteriormente definido. Éste, es decir $U$, es el género próximo, y $\mathrm{P}$ es la diferencia específica.

El que existan conjuntos construibles y no construibles parece contradecir lo que afirma Zubiri, respecto a que todo lo matemático está construido, «todo objeto está construido postuladamente» (Zubiri, 1982, p. 138). Pero esta contradicción es sólo aparente porque lo que Zubiri llama construcción es algo distinto a lo que así llaman Gödel y Cohen. Zubiri nos dice: "Lo que Gödel y Cohen construyen es en el fondo el concepto objetivo tanto específico como genérico» (Zubiri, 1982, p. 137). Mientras que, para el filósofo español, la construcción consiste en realizar ante la inteligencia un concepto ya constituido objetivamente (ya sea construible o no construible según Gödel y Cohen, respectivamente). En consecuencia, la construcción en Zubiri es algo muy distinto de la construcción a la Gödel y Cohen. De acuerdo al modo zubiriano de construcción, tanto los conjuntos construibles de Gödel, como los no construibles de Cohen están construidos, en el sentido de realizados ante la inteligencia. La construcción zubiriana es la realización de un contenido en la realidad física, es una realización intelectivamente libre en «la» realidad.

De acuerdo al concepto de construcción de Zubiri, los conjuntos de Gödel y Cohen no sólo están construidos como conceptos objetivos específicos y genéricos, sino que también están construidos en «la» realidad física, de lo cual se sigue, que la construcción a la Zubiri no concierne formalmente a los conceptos, no es "conceptiva», sino que se trata de una realización en «la» realidad física "según conceptos». Se trata de una realización ante la inteligencia de un concepto que ya ha sido construido objetivamente, mientras que la construcción tanto en Gödel como en Cohen, sería algo puramente conceptivo. La construcción zubiriana requiere previamente de la construcción objetiva tanto específica como genérica del concepto antes de proyectar lo irreal del concepto sobre «la» realidad «según conceptos». Por ello los matemáticos hablan en modo indicativo y no potencial cuando se refieren a los objetos matemáticos.

\section{LA CONSTRUCCIÓN EN EL SENTIDO DE ZUBIRI V/S LA CONSTRUCCIÓN EN EL SENTIDO DE BROUWER (INTUICIONISMO)}

Zubiri también delimita y distingue su concepto de construcción del de los intuicionistas, en particular el de Brower. Veremos a continuación en qué difieren estos conceptos.

Frente a la crisis de los fundamentos de las matemáticas y sus consecuencias en relación con el estatuto epistemológico de esta ciencia, el intuicionismo de Brouwer fue el que se opuso con mayor fuerza y rigor al formalismo axiomático de Hilbert. Frente al formalismo, el intuicionismo considera que las colecciones infinitas son las causantes de las paradojas que emergieron en el seno de la teoría de conjuntos, además se opone al método de reducción al absurdo que se usa la mayoría de las veces que se quiere probar algo en relación con este tipo de colecciones, y termina oponiéndose y no aceptando los principios de no contradicción y tercero excluido, entre otras técnicas, métodos y principios que considera ilegítimos. Esta postura radical del intuicionismo lo llevó a sólo poder aceptar, como legítimas, colecciones finitas de elementos, y a lo sumo, colecciones infinitas contables de elementos. Esto condujo a los intuicionistas a un finitismo incompatible con gran parte del análisis, lo cual a su vez, los llevó a aceptar sólo aquellos objetos y operaciones matemáticas que fueran construibles por medio de métodos finitistas. El intuicionismo al rechazar los principios lógicos fundamentales de no contradicción y tercer excluido, rechaza la idea de que las matemáticas se funden en la lógica. En efecto, una demostración que se funde en los principios lógicos de no contradicción y tercer excluido no es para Brouwer una demostración matemática. Entonces, para el intuicionismo construir matemáticamente no es lo mismo que definir y construir conceptos, la matemática no es un sistema de conceptos y operaciones definidas. Para el intuicionismo una operación es matemática si se puede ejecutar en un número finito de pasos. Pero gran parte de la matemática moderna y sobre todo el análisis, se ocupan de conjuntos infi- 
nitos no contables, como por ejemplo: los números reales y complejos; la representación decimal de números trascendentes tales como pi, e, omega, etc.; las soluciones de EDP o ecuaciones integrales; el conjunto de Julia u otros conjuntos fractales; etc.

En matemáticas no se pueden ejecutar todas las operaciones necesarias para, por ejemplo, obtener todos los decimales de pi. Peor aún existen números reales de complejidad infinita, como por ejemplo el número omega descubierto por G. Chaitin. Para calcular este número no existe un algoritmo que tenga un número de «bits» considerablemente más pequeño que el número de decimales de omega a calcular. En otras palabras, el número omega no es comprimible por medio de una fórmula que nos permita calcular sus decimales de manera indefinida, como de hecho sí sucede con, por ejemplo, los números trascendentes «pi» y «e». Sin embargo, esto no quita que puedan seguir creándose nuevos métodos, nuevas formas de pensar, que permitan resolver este tipo de problemas. La matemática, en general, y el análisis, en particular, continúan en la búsqueda de nuevos métodos y formas de pensar la realidad problemática de las matemáticas relacionada con el infinito no contable y gran parte de su cuerpo de conocimiento dice relación con conjuntos infinitos. Por lo tanto, el finitismo de Brouwer es inaceptable para los matemáticos en general, porque se tendría que eliminar un enorme pedazo del edificio matemático. Pero dejemos esto de lado, porque, por una parte, no contribuye a entender más claramente el concepto de construcción zubiriano, y por otra parte, obviamente nos aleja del tema principal, a saber: ¿cuál es la diferencia entre el concepto de construcción de Brouwer y de Zubiri?

Lo que tenemos que entender en el presente apartado es que el intuicionismo se opone al formalismo con un concepto de construcción diferente al de este, y que no debe confundirse con el de Zubiri. En este sentido, el intuicionismo opone al formalismo un finitismo, que se traduce en la exigencia de que toda operación debe ser ejecutada en un número finito de pasos y que Zubiri considera insostenible: «porque ni los conjuntos [por finitos que sean], son formalmente intuitivos, ni las operaciones ejecutadas sobre ellos constituyen lo radical de lo que yo entiendo por construcción matemática» (Zubiri, 1982, p. 140).

Oponiéndose a todos los exponentes y defensores de la así llamada "Teoría Intuitiva de Conjuntos», entre los cuales se encuentran Brouwer, como fundador, y Paul R. Halmos (1965) como continuador, Zubiri nos dice: «el conjunto finito de Brouwer no es intuitivo [...] intuición es la "visión" de algo dado inmediatamente, directamente, unitariamente. En la intuición tengo la diversidad cualitativa y cuantitativa de lo dado, pero nunca tengo un conjunto. No hay estrictos conjuntos intuitivos. Porque para tener un conjunto necesito considerar aisladamente, por así decirlo, los momentos de la diversidad intuitiva como "elementos". Sólo entonces su unidad constituye un conjunto» (Zubiri, 1982, pp. 140-141). Es difícil argumentar contra Zubiri en esto, porque para él prácticamente por definición nunca se tiene un conjunto en la intuición. En la intuición se podrá tener la diversidad cualitativa y cuantitativa de algo dado, pero nunca un conjunto, porque: «conjunto matemático es siempre y sólo conjunto de elementos. Pero entonces es claro que ningún conjunto, ni tan siquiera finito, es intuitivo» (Zubiri, 1982, p. 141). Aquí tenemos algunos problemas, porque esta última afirmación de Zubiri es cuestionable. Para ello basta considerar el conjunto vacío, los singleton (conjuntos de un solo elemento), y el conjunto universo, los cuales son usados intensamente en matemáticas y ciertamente en teoría de conjuntos. De hecho, la propia teoría de conjuntos no sería una teoría si no considerara los conjuntos mencionados. Ahora y por cierto, si nosotros descartamos los conjuntos mencionados como conjuntos legítimos, desde el punto de vista del sentido común, Zubiri tendría razón, aunque desde el punto de vista matemático, y sobre todo desde el punto de vista axiomático, son conjuntos legítimos.

Dejando a un lado el problema planteado por los conjuntos antes mencionados, podemos decir junto con Zubiri que los conjuntos finitos de Brouwer, presuntamente dados en la intuición, requieren de una construcción ante la inteligencia que reúna la diversidad de momentos dados en la intuición. "La intuición no da sino "diversidad de momentos", pero jamás nos da "conjuntos de elementos". Para tener un conjunto es necesario un acto ulterior de intelección que haga de los momentos elementos. Hace falta pues una construcción» (Zubiri, 1982, p. 141). Entonces, el conjunto finito de Brouwer es: «la aplicación del conjunto ya construido intelectivamente a la diversidad de lo dado» (Zubiri, 1982, p. 141), y esta aplicación es un ejemplo de lo que Zubiri ha llamado previamente, en general, construcción por postulación. En este caso particular: "se postula que lo dado se resuelve en un conjunto» (Zubiri, 1982, p. 141), y Zubiri concluye esta parte de su argumentación diciendo: «Por consiguiente, en estricto rigor no puede llamarse intuicionismo a la matemática de Brouwer. El conjunto de Brouwer no es intuitivo; es el contenido objetivo de un concepto de conjunto que se "aplica" a lo intuitivo» (Zubiri, 1982, p. 141), es decir, a los diversos momentos dados en la intuición. 
No contento con lo anterior, Zubiri nos da un segundo argumento contra el intuicionismo de Brouwer para aclarar la diferencia entre su concepto de construcción y el de Brouwer, diciéndonos: «En segundo lugar, la construcción misma del conjunto no es radicalmente un sistema de operaciones ejecutadas. Digo "radicalmente", porque la ejecución de operaciones no es lo primario de lo que he llamado construcción" (Zubiri, 1982, p. 141). Por supuesto que no, porque para Zubiri construir, recordémoslo, es principalmente proyectar lo irreal de los conceptos sobre «la» realidad «según conceptos». Por ello que nuestro autor nos dice a reglón seguido: «El conjunto finito es contenido de conceptos objetivos. Por esto las operaciones ejecutadas sobre este contenido son operaciones, todo lo ejecutadas que se quiera, pero siempre ejecutadas sobre contenidos objetivos de conceptos. Finitos o no, los conjuntos de que se ocupa la matemática de Brouwer y las operaciones sobre ellos ejecutadas son conjuntos y operaciones conceptivas» (Zubiri, 1982, p. 141). En consecuencia, todavía no son reales, siendo necesarias, no son suficientes para fundamentar lo matemático, porque como ya ha sido dicho previamente por nuestro autor y lo vuelve a repetir ahora: "la matemática no trata de "conceptos objetivos" sino de "cosas que son así"» (Zubiri, 1982, p. 142). Son así, por postulación.

Lo que Zubiri entiende por construcción es muy distinto de lo que entienden Gödel, Cohen y Brouwer. En efecto, para Zubiri: "construir no es ejecutar operaciones objetivas [ni definir axiomáticamente] sino proyectar ante mi inteligencia ese contenido objetivo en "la" realidad física. Y esta realidad no está dada en intuición sino en aprehensión primordial de realidad; está dada impresivamente. Como esta realidad no tiene contenido determinado yo puedo proyectar libremente sobre ella el contenido de lo objetivamente construido operacionalmente. Esta proyección y no la operación es la construcción matemática. El objeto matemático, aunque sea finito, y aunque la operación que objetivamente produzca su contenido sea ejecutada, tiene sin embargo una radical realidad propia, la realidad física impresivamente sentida en aprehensión primordial» (Zubiri, 1982, p. 142). Esto es construcción para Zubiri.

Resumiendo lo dicho hasta ahora, podemos decir que para Zubiri construir no consiste en estar definido en el sentido de Gödel y Cohen, tampoco es estar ejecutado en el sentido de Brouwer, sino que es otra cosa: "es crear, es proyectar libremente en "la" realidad física un contenido según conceptos. Postular es postular realidad. Sin esta construcción y postulación radical y primaria serían imposible tanto los axiomas de Zermelo-Frankel y los conjuntos de Cohen como el intuicionismo de Brouwer» (Zubiri, 1982, p. 143).

\section{SOBRE LA INTERPRETACIÓN ZUBIRIANA DE LOS TEO- REMAS DE GÖDEL Y COHEN: COMENTARIO Y CRÍTICA}

Antes de comentar las ideas de Zubiri respecto a los teoremas de Gödel y Cohen, digamos primero algo respecto a estos teoremas. El teorema de Gödel emerge después de una larga historia, que comienza con G. Cantor (1845-1918) y su definición intuitiva de conjunto, la cual, por una parte, motivó la paradoja de B. Russell (1872-1970), interpósito el trabajo de G. Frege (1848-1925), y por otra parte, generó una crisis en los fundamentos de las matemáticas, en el estatuto epistemológico de esta ciencia. Una de las reacciones frente a esta falta de fundamento, además de los programas logicista e intuicionista, fue el programa formalista propuesto por D. Hilbert (1862-1943), el cual consistía en reducir toda la matemática, si no todo el conocimiento humano, a un conjunto de axiomas consistente y completo. El teorema de Gödel vino a darle en el traste al sueño hilbertiano, al demostrar que cualquier sistema axiomático suficientemente rico, como para que contenga la aritmética clásica (es decir, los axiomas de G. Peano (1858-1932)), es inevitablemente incompleto, es decir, que existen proposiciones que siendo verdaderas no son demostrables o deducibles dentro del sistema axiomático en cuestión. Dicho de otra manera, el árbol deductivo asociado al sistema axiomático en examen tiene hoyos inaccesibles por medio de la "escalera deductiva», o lo que es lo mismo, existen proposiciones que son indecidibles dentro del sistema axiomático a la mano, diferenciando de una vez y para siempre las nociones de verdad y demostrabilidad (Nagel y Newman, 2007).

El teorema de P. J. Cohen (1963), por su parte, dice relación con la hipótesis del continuo enunciada por G. Cantor en el contexto de su teoría de conjuntos transfinitos. Según esta hipótesis no existe otro infinito entre el infinito contable (cardinalidad de los números naturales) y el continuo (o cardinalidad de los números reales). En otras palabras, el infinito que sigue después del infinito contable es el continuo. K. Gödel logró demostrar en 1938 que la hipótesis del continuo de Cantor (en su versión generalizada) era consistente con los axiomas de la teoría de conjuntos de Zermelo-Fraenkel (lo cual visto parcialmente, es decir independientemente de otros resultados, diría que la hipótesis del continuo de Cantor sería verda- 
dera, si consideramos los axiomas de Z-F verdaderos). P. J. Cohen, para "agregarle más flecos a la colcha», o «mayor abundamiento», demostró en 1963 que la negación de la hipótesis del continuo (en su versión generalizada) también era consistente con los axiomas de la teoría de conjuntos de Zermelo-Fraenkel, lo cual visto parcialmente, es decir independientemente del resultado de K. Gödel mencionado más arriba, diría que la hipótesis del continuo de Cantor sería falsa, si consideramos los axiomas de Z-F verdaderos. ¿Quiere decir esto que tenemos un matemática cantoriana (en la cual es válida la hipótesis del continuo de Cantor), y una matemática no-cantoriana, donde por el contrario no es válida la hipótesis del continuo de Cantor, a la manera en que coexisten la geometría euclidiana y las geometrías no euclidianas? Quizá la cuestión no sea tan simple y lo que falte sean desarrollos adicionales. Como afirma K. Gödel (mucho antes que $X$. Zubiri) refiriéndose a los axiomas de la teoría de conjuntos: "[Éstos] describirían alguna realidad bien determinada en la cual la conjetura de Cantor debería ser cierta o falsa. Por ello su indecidibilidad a partir de los axiomas que hoy día aceptamos [axiomas Z-F] sólo puede significar que estos axiomas no entrañan una descripción completa de esta realidad» (Gödel, 2006, p. 362). El teorema de Cohen no demuestra, por tanto, la falsedad de la hipótesis del continuo de Cantor (HCC), como afirma Zubiri (1982, p. 137) ${ }^{4}$, sino que junto con el trabajo de Gödel de 1938, demuestra su indecidibilidad, es decir, la imposibilidad de decir si es verdadera o falsa en el contexto de los axiomas de Z-F.

Según Zubiri, los resultados de estos teoremas vendrían a mostrar la anterioridad de la realidad sobre la verdad ${ }^{5}$. Creemos que esto equivale a decir derechamente (siendo más precisos y coherentes con las observaciones que haremos a continuación), que la realidad en la ciencias matemáticas es anterior a la lógica, o a la consistencia, o al principio de no contradicción. En efecto, si las construcciones matemáticas no son meros conceptos, si construir no es sólo hacer de algo término intencional e irreal, sino que consiste en proyectar esto irreal del concepto sobra "la» realidad "según conceptos»; entonces construir por postulación es un modo de realizar antes que establecer la «verdad», "lo verdadero» estará por verse en un segundo momento. Consistentemente con esto, Zubiri nos dice: «El teorema de Gödel, según el cual lo construido por postulación tiene "de suyo" más propiedades que las formalmente postuladas, expresa a mi modo de ver que lo postulado es realidad antes que verdad» (Zubiri, 1982, p. 145).
El teorema de Gödel (1931), visto desde un punto de vista estrictamente técnico, entre otras consecuencias, separa de una vez y para siempre las nociones de verdad y demostrabilidad (Gödel, 1981, p. 53). Es decir, todos los teoremas demostrables podrán ser «verdaderos» (si los axiomas a partir de los cuales se deducen son "verdaderos», o son "considerados verdaderos»), pero no todo lo "verdadero" es demostrable. El teorema de Gödel al separar las nociones de verdad y demostrabilidad, nos coloca en la situación de preguntarnos si acaso la noción de verdad es una noción lógica, lo cual pareciera que es negativo, o si la verdad es a veces es real y otras veces es lógica. Si la noción de verdad no es real, ni lógica: ¿qué es?, ¿qué papel o rol desempeña entre realidad y demostrabilidad?, ¿será la noción de verdad un concepto meramente regulador, que de acuerdo al pensamiento formalista valdría más sustituir por los conceptos de consistencia y completitud? De hecho, una de las consecuencias del teorema de Gödel para aquellos sistemas axiomáticos que contengan al menos los axiomas de Peano es que, o son consistentes, o son completos, pero no pueden tener ambas propiedades a la vez, y en matemáticas, por la naturaleza propia de la disciplina, se opta por la consistencia (es decir, por el principio de no contradicción), donde la noción de verdad no juega ningún papel (o mejor dicho, se despeja al comienzo adoptando el principio de no contradicción en su lugar). Según esto, más que decir que el teorema de Gödel expresa que lo postulado es realidad antes que verdad, como hace Zubiri, mejor sería decir que este teorema expresa que lo postulado es real antes que "lógico o consistente» ${ }^{6}$. Por otro lado, Zubiri tiene razón cuando afirma que la incompletitud de este tipo de sistema axiomático, como dijo Gödel en 1947: "solamente puede significar que esos axiomas no contienen una descripción completa de esa realidad» (Gödel, 2006, p. 362). Nunca la tendrán podríamos agregar nosotros, y en consecuencia, viene a colocar en evidencia que el método axiomático no es el adecuado para describir los objetos matemáticos, o que nunca seremos capaces de tener una descripción consistente y completa de objetos como por ejemplo los así llamados conjuntos.

Sobre el teorema de Cohen, según el cual la negación de la HCC también es consistente con los axiomas de Z-F (donde Cohen utiliza la noción de conjuntos genéricos ya mencionada y discutida antes), Zubiri nos dice: «los conjuntos no son sólo sistemas de elementos determinados por precisa postulación, sino que hay, antes de eso, conjuntos que él [Cohen] llama genéricos y que a mi modo de ver no son genéricos, 
sino que son la simple realización del conjunto, sin las propiedades específicas determinadas por postulación» (Zubiri, 1982, pp. 145-146). Puesto que todo conjunto tiene propiedades genéricas y específicas, lo que dice Zubiri en esta cita es que el concepto de conjunto genérico no alude tan sólo a lo que nosotros, y tal vez Cohen, podríamos llamar el género próximo de una definición, sino que también alude a la realización por postulación de un conjunto, a la afirmación de la existencia postulada de un conjunto, al conjunto como realidad postulada, al género próximo como realidad postulada. Es decir, los conjuntos genéricos de Cohen, según Zubiri, son el género próximo de una definición realizada por postulación ante la inteligencia, pero sin las propiedades específicas del objeto así postulado (las cuales por lo demás, también son realizadas por postulación). Entonces, nos dice Zubiri: «La especificación no es aquí una diferencia lógica sino una determinación real» (Zubiri, 1982, p. 146). En consecuencia, siguiendo a Zubiri, se puede inferir que las propiedades postuladas de los conjuntos en los axiomas son reales antes que "verdaderas», o lo que es lo mismo: "la realidad del conjunto [en lo que respecta a sus notas o propiedades genéricas y específicas] está antes que la verdad [consistencia lógica] axiomáticamente postulada» (Zubiri, 1982, p. 146). Por ello que Zubiri termina el apéndice en comento, diciendo a modo de conclusión: "A mi modo de ver, éste es el sentido esencial de los teoremas de Gödel y Cohen: la anterioridad de lo real sobre lo verdadero [la lógica o consistencia o principio de no contradicción] en la matemática» (Zubiri, 1982, p. 146).

Ahora bien, el teorema de Gödel no es un resultado universal. En primer lugar, dentro de las ciencias matemáticas existe una variedad de métodos diferentes al axiomático, y el teorema de Gödel sólo ha venido a probar el fracaso de éste método en cuanto y en tanto es imposible obtener una formalización consistente y completa de ciertos objetos matemáticos que son tratados como primitivas dentro del sistema formal. Pero no está dicho, y menos probado, que otro método no pueda lograr una definición satisfactoria, si no de todos, al menos de algunos objetos matemáticos. En segundo lugar, existen una gran variedad de sistemas axiomáticos consistentes y completos en matemáticas (por ejemplo, el cálculo de predicados de primer orden, la geometría de Hilbert, etc.) en los que no se cumple el teorema de Gödel, y en los que, podríamos decir, que lo lógico o la consistencia o el principio de no contradicción están a la misma altura que lo real, o antes que lo real. En definitiva, si bien es cierto, que el teorema de Gödel y sus consecuen- cias deben ser tomadas en serio desde el punto de vista de cualquier teoría del conocimiento, debemos notar que no se trata de un resultado de aplicación universal, ni siquiera dentro de las ciencias matemáticas. El teorema de Gödel sólo es válido para el método axiomático y dentro de éste sólo para aquellos sistemas axiomáticos lo suficientemente ricos como para que contengan los axiomas de Peano (o de la aritmética clásica). En consecuencia, a partir de los teoremas de Gödel y Cohen no podemos sacar la conclusión general de que: lo real esté antes que lo verdadero en la matemática.

Dejando a un lado aquellos objetos matemáticos que no sean formalizables por medio de un sistema axiomático consistente y completo, o lo que es lo mismo, para aquellos objetos matemáticos para los que sea válido el teorema de incompletitud de Gödel, podemos decir junto a Zubiri, matizando lo que dice: «Por esto y sólo por esto es por lo que [el tipo de objeto matemático que no puede ser formalizado por medio de un sistema axiomático consistente y completo] plantea problemas que pueden no ser resolubles con el sistema finito de axiomas y postulados que han definido su realización. Lo [así] construido en "la" realidad es, por estar realizado [de esa manera], algo más que lo postulado al realizarlo. Es a mi modo de ver el alcance del teorema de Gödel» (Zubiri, 1982, p. 138). Así las cosas, para Zubiri, el teorema de Gödel, no sólo coloca en evidencia las limitaciones del método axiomático (lo cual es sabido desde la aparición de este teorema), sino que además, y lo que es más importante: «deja al descubierto ante la inteligencia el carácter de realidad de lo construido según los axiomas y postulados en cuestión» (Zubiri, 1982, p. 139). Al igual que para Gödel (2006, p. 362), la incompletitud no sería para Zubiri un asunto de limitación o incapacidad en el conocimiento humano, sino que una característica propia de objetos reales, que en tanto reales son «más» que un contenido determinado ${ }^{7}$.

Antes de terminar, es necesario llamar la atención sobre un hecho que Zubiri no menciona, ni se ocupa de los sistemas completos y consistentes, sistemas, que a nuestro juicio, ponen en entredicho considerablemente su postura ontológica frente a los objetos matemáticos, y sobre todo su interpretación de los teoremas de Gödel y Cohen, restándole universalidad a sus afirmaciones. Porque si aplicamos las ideas de Zubiri a sistemas axiomáticos consistentes y completos, habría que concluir que los objetos formalizados por tal tipo de sistemas no tienen un «más», o no dan «más de sí», porque estarían completamente caracte- 
rizadas sus notas (o propiedades), su existencia, y su contenido por tal sistema axiomático. En consecuencia, no serían reales, de todo lo cual se sigue que las matemáticas tendrían objetos reales y objetos que no serían reales en el sentido zubiriano. Entonces no todos los objetos matemáticos serían reales por postulación. Por otra parte, ¿qué hay de aquellos objetos matemáticos que no se presentan bajo sistema axiomático alguno, es decir, qué hay de aquellos objetos matemáticos que no son postulados por sistema axiomático alguno? Estas preguntas quedan aún abiertas, creemos, a partir de y en la filosofía de Zubiri.

\section{CONCLUSIONES}

Después de revisar exhaustivamente el apéndice "La realidad de lo matemático», es claro que el realismo matemático de Zubiri ${ }^{8}$, que quizás podríamos llamar "constructivismo sentiente» es muy distinto de otros realismos y posiciones filosóficas respecto a la matemática y sus objetos ${ }^{9}$. Su idea de que la matemática es un sistema de juicios o verdades acerca de objetos reales, reales por postulación o construcción, se distancia de la posición tan común entre algunos matemáticos que considera a los objetos matemáticos como ideales. Por otra parte, la manera en que comprende Zubiri la "construcción» es distinta de la de los formalistas o intuicionistas, pues para el filó- sofo español se trata de postulación o construcción de un contenido en "la» realidad y no meramente de una construcción conceptiva (ya sea deducción o ejecución de operaciones).

Respecto a la interpretación zubiriana de los teoremas de Gödel y Cohen, sólo cabe repetir dos puntos ya mencionados. En primer lugar, el teorema de Cohen no demuestra la falsedad de la Hipótesis del Continuo de Cantor como afirma Zubiri (Zubiri, 1982, p. 137), sino que complementado con el teorema de Gödel, viene a probar la indecibilidad de la HCC en el contexto de los Axiomas de Z-F. En segundo lugar, es necesario matizar la afirmación de Zubiri respecto a que los teoremas de Gödel y Cohen mostrarían «la anterioridad de lo real sobre lo verdadero en la matemática» (Zubiri, 1982, p. 146), puesto que el alcance de estos teoremas dista de ser universal, incluso dentro de las matemáticas, y por otra parte, hay que tener en cuenta, que existen objetos matemáticos en sistemas axiomáticos consistentes y completos que no cumplirían la noción de objeto matemático real de Zubiri.

\section{AGRADECIMIENTOS}

Este trabajo ha sido financiado gracias al proyecto FONDECYT (Chile) N 1141260 y a la Universidad Técnica Federico Santa María.

\section{NOTAS}

1. Traducción de los autores. Aquí el texto original: "Mathematicians, though privy to a wider range of mathematical truths than most of us, often incline to agree with unsullied common sense on the nature of those truths. They see themselves and their colleagues as investigators uncovering the properties of various fascinating districts of mathematical reality: number theorists study the integers, geometers study certain well behaved spaces, group theorists study groups, set theorists sets, and so on".

2. Algunas definiciones tradicionales de juicio: juicio es el acto mental por medio del cual nos formamos una opinión de algo; juicio es el proceso mental por medio del cual decidimos conscientemente que algo es de un modo u otro; juicio es la afirmación o la negación de algo (de un predicado) con respecto a algo (un sujeto); juicio es un acto mental por medio del cual se une (o sintetiza) afirmando, o se separa negando; juicio es una operación de nuestro espíritu en la que se contiene una proposición que es o no conforme a la verdad y según la cual se dice que el juicio es o no correcto; juicio es un producto mental enunciativo; juicio es un acto mental por medio del cual pensamos un enunciado; juicio es un acto del entendimiento basado en la fuerza de convicción; juicio es el conocimiento mediato de un objeto; juicio es la facultad de juzgar o también el resultado de la facultad de juzgar.

3. Respecto a la idea de Zubiri de considerar tanto los objetos matemáticos como los personajes novelas como reales, sería interesante hacer estudios e investigaciones para ver en qué medida el realismo matemático de Zubiri podría constituir una respuesta al ficcionalismo de Hartry Field (1989).

4. Esta afirmación de Zubiri, sin embargo, no afecta en lo esencial sus argumentos respecto a la realidad por postulación de los objetos matemáticos, y tampoco afecta esencialmente la interpretación que hace de los teoremas de Gödel y
Cohen, en el sentido de que lo real está antes que la verdad en matemáticas, siempre y cuando estemos hablando de objetos, o aquellas partes de la matemática, que no sean formalizables por medio de un sistema axiomático consistente y completo.

5. Respecto a la relación realidad y verdad: «No es idéntico realidad y verdad porque hay o puede haber realidades que no estén actualizadas ni tienen por qué estarlo. En este sentido no toda realidad es verdad. Verdad es una cualidad de la actualización, y la actualización es un momento físico de lo real: sin añadirle ninguna nota más, la actualización sin embargo añade a las notas realmente la verdad. Por esto, verdad y realidad no sólo no son idénticas, sino que tampoco son meramente correlativas: realidad no es mero correlato de la verdad sino fundamento de ésta, porque toda actualización lo es de realidad. La realidad es pues lo que da verdad a la intelección, es lo que "verdadera" en ella» (Zubiri, 1982, p. 255). 
6. Ahora bien, hilando más fino, y equiparando lo "verdadero» con: la lógica, la consistencia y el principio de no contradicción, como lo hemos insinuado antes, y puesto que: el principio de identidad, el principio de no contradicción y el principio del tercero excluido son inseparables y dicen más o menos lo mismo; podríamos inferir que lo que dice el teorema de Gödel (para los casos en que sea aplicable y en sus propios términos), es que si queremos mantener la identidad, la no contradicción, y un solo ser del objeto así formalizado, tenemos que pagar el precio de no poder admitir: el movimiento, el cambio, la evolución, la generación, la degeneración, la negación de sí mismo, la vida, etc. Entonces, contraviniendo lo dicho por Zubiri podríamos decir: «no se trata de que lo real esté antes que lo "verdadero" en la matemática, sino que lo real no está en la matemática». Mejor dicho: «no se trata de que lo real esté antes que lo "verdadero" en la formalización axiomática de la matemática, sino que lo real no está, no puede estar en dicho tipo de formalización, sin perjuicio de que podría estar en otra forma de hablar (describir, representar, formalizar, etc.) de los objetos matemáticos».

7. Alan Turing (1912-1954) habría estado de acuerdo en que la incompletitud es una limitación humana y no meramente lógica, pero Turing llegó a esta conclusión por una razón diferente. En efecto, Turing pensaba que el ser humano era una máquina y puesto que él mismo había probado que existen problemas no computables en las matemáticas (su famoso problema de la parada: «no existe un programa que prediga cuando un programa dado se va a detener o no», de hecho si uno supone que un tal programa existe llega a una contradicción, por lo tanto su demostración es por reducción al absurdo), se sigue que el ser humano es inherentemente limitado en su naturaleza (Turing, 1937). Sin embargo, Gödel a diferencia de Turing, pensaba que el ser humano no se reducía a una máquina, sino que tenía una chispa divina, y lo que no lograba capturar o aprehender lógicamente, lo haría por medio de la intuición y la creatividad. Durante su estadía en el Instituto de Estudios Avanzados de Princeton (USA), y después de intentar probar la «Hipótesis del Continuo» de Cantor sin éxito, Gödel intentó probar la existencia de la intuición y la creatividad por medios lógicos y como una alternativa al método lógico, lo cual es obviamente paradójico. En este contexto problemático, Gödel va perdiendo la razón lentamente y se suicida dejando de comer. ¿Cómo definir y/o probar la existencia de la intuición, la creatividad, la conciencia y la vida?; ¿es el ser humano una máquina o no? Los adherentes al programa fuerte de la inteligencia artificial trabajan arduamente bajo la hipótesis de que el pensamiento y el cerebro humano son reducibles a una máquina; mientras que Roger Penrose piensa que no es así, y en su libro titulado The Emperor's New Mind (1989) usa el Teorema de Incompletitud de Gödel para defender su posición. Greg Chaitin está trabajando en post de alcanzar una definición de creatividad, para ello está sentando las bases de una nueva área del conocimiento, que él llama «Metabilogía», la cual sería un campo paralelo a la bilogía, que estudia la evolución por medio de mutaciones aleatorias de "software» artificiales, de manera que se puedan demostrar teoremas o por lo menos avanzar argumentos heurísticos al mismo alto nivel que se acostumbra a hacer en la física teórica, para demostrar o argumentar a favor de la creatividad en las matemáticas y la biología, entre otros objetivos muy difíciles de lograr; sin embargo ya tiene algunos resultados preliminares muy auspiciosos, que se encuentran en su "pre-print» titulado "To a mathematical theory of evolution and biological creativity» (2011). Willard L. Miranker y Gregg J. Zuckerman (2008), que trabajan en el Departamento de Ciencias de la Computación de la Universidad de Yale (USA), están trabajando en los fundamentos matemáticos de la conciencia, y para ello se valen de los axiomas de Zermelo-Fraenkel para la teoría de conjuntos y un axioma que ellos llaman de anti-fundamento.

8. Quizá podríamos considerarlo, en cierto sentido, cercano, o al menos no irreconciliable al realismo naturalista encarnado de Lakoff y Nuñez (2000), y la matemática como ciencia de las estructuras fundamentales de la realidad propugnada por quien escribe.

9. Algunas posiciones filosóficas destacadas respecto a las matemáticas son: al formalismo hilbertiano; el logicismo fregianorusselliano; el intuicionismo de Brouwer; el idealismo platónico; el ficcionalismo de Hartry Field, quien rechaza e invierte el argumento de indispensabilidad de Quine, sugiriendo que la matemática es dispensable, y por lo tanto, es un cuerpo de falsedades que no habla acerca de nada real, sino que es una ficción útil. Algunas posiciones realistas: el realismo sensual aristotélico, en el sentido de que a las ideas en general y a los conceptos o ideas matemáticos en particular, se llega por medio de un proceso de abstracción y descontextualización delirante en que van quedando atrás las cualidades sensi- bles de los objetos; el realismo ingenuo, en el sentido de que existe algo bien definido independientemente de la acción y el pensamiento humanos $y$, sin embargo, capaz de ser descrito adecuadamente en un lenguaje humano, porque tenemos acceso directo a ello a través de la aprehensión; el realismo pragmático, en el sentido de que tomamos las cosas u objetos como cosas reales tal cual se nos presentan en la práctica diaria (intrínsecamente vinculadas a la práctica de la vida); la de un realismo naturalista encarnado a la Lakoff y Núñez, que a partir de las ciencias cognitivas modernas ven la matemática como un conocimiento enraizado en la encarnación cerebral de la mente y de la experiencia diaria, y tratan de explicar cómo las ideas matemáticas emergen en nuestros cuerpos y cerebros a través de mecanismos cognitivos claramente formulables, tales como las metáforas conceptuales, las cuales importan modos de razonamiento de la experiencia senso-motora y, en consecuencia, las matemáticas no existirían en ningún sentido real, excepto en el cerebro humano; el realismo de Penelope Maddy, que a partir del argumento de indispensabilidad de Quine (el cual establece que las matemáticas son el lenguaje fundamental e indispensable de nuestras mejores teorías científicas, particularmente de la física, y que por lo tanto deben ser aceptadas como un cuerpo de conocimiento verdadero, que habla acerca de entidades existentes independientemente), entre otros argumentos, infiere que los objetos matemáticos deben ser tan reales como los objetos de la física; la posición realista de quien escribe, que piensa que por debajo de nuestro mundo operan las leyes de la ciencia, y que por debajo de éstas operan leyes aún más profundas, a saber leyes matemáticas, es decir las matemáticas como la ciencia de las estructuras fundamentales de la realidad; el empirismo, que niega que las matemáticas se puedan conocer a priori de ninguna manera, afirmando que nosotros descubrimos los hechos matemáticos a través de investigaciones empíricas; el quasi-empirismo de inspiración Popperiana y desarrollado por Imre Lakatos, Greg Chaitin y Stephen Wolfram, que dice que los matemáticos en su investigación, por una parte, proponen y testean hipótesis, y por otra, demuestran teoremas, porque un argumento matemático puede transmitir falsedad de las conclusiones a las premisas, como así mismo transmitir verdad de las premisas a la conclusión; el realismo social o constructivismo social, que ve las matemáticas primariamente como una construcción social, como un producto de la cultura, sujeto a correcciones y cambios; o etc. 


\section{BIBLIOGRAFÍA}

Cohen, P. J. (1963). The independence of the continuum hypothesis. Proceedings of the National Academy of Sciences of the U.S.A, 50, 6, pp. 1143-1148. http:// dx.doi.org/10.1073/pnas.50.6.1143

Chaitin, G., (2011). To a mathematical theory of evolution and biological creativity. [En línea]. [Fecha de consulta: 18 de julio de 2014]. Disponible en: https://www. cs.auckland.ac.nz/ chaitin/darwin.pdf.

Field, H. (1989). Realism, Mathematics and Modality. Blackwell.

Gödel, K. (2006). Obras completas. Madrid: Alianza.
Halmos, P. R. (1965). Teoría intuitiva de los conjuntos. Ciudad de México: Continental.

Lakoff, G. y Núñez, R. E. (2000). Where Mathematics comes from. New York: Basic Books.

Maddy, P. (1990). Realism in Mathematics. Oxford: Clarendon Press.

Miranker, W. y Zuckerman, G. J. (2008) Mathematical Foundations of Consciousness. [En línea]. [Fecha de consulta: 18 de Julio de 2014]. Disponible en: http://arxiv.org/ftp/arxiv/ papers/0810/0810.4339.pdf.
Nagel, E. y Newman, J. R. (2007). El teorema de Gödel. Madrid: Tecnos.

Penrose, R. (1989). The Emperor's New Mind: Concerning Computers, Minds, and The Laws of Physics. New York: Oxford University Press.

Turing, A. (1937). On computable numbers, with an application to the Enstcheidungsproblem. Proceedings of the London Mathematical Society, 42, pp. 230265. http://dx.doi.org/10.1112/plms/ s2-42.1.230

Zubiri, X. (1982). Inteligencia y Logos. Madrid: Alianza. 